\title{
Use of Patterns for Knowledge Management in the Ceramic Tile Design Chain
}

\author{
María Jesús Agost ${ }^{1}$, Fernando Romero $^{1}$, Carlos Vila $^{1}$ and Pedro Company ${ }^{2}$ \\ CINEI (Industrial Enterprise Innovation Center. Universitat Jaume I. Campus del Riu Sec. E12071 Castellón. Spain. \\ ${ }^{1}$ Industrial Systems Engineering and Design Department \\ magost@guest.uji.es; \{romero, vila\}@esid.uji.es \\ ${ }^{2}$ Mechanical Engineering and Construction Department \\ pcompany@.uji.es
}

\begin{abstract}
Knowledge Management (KM) is a complex objective, especially in the instance of extended enterprises consisting of SMEs, and critical in new product design and development (NPD). The use of patterns is essential to get KM in collaborative NPD processes. This paper presents the use of patterns adopted in the CE-TILE project to standardize information and knowledge in collaborative work. The different types of patterns and models established for the knowledge capture, formalization and configuration are also described.
\end{abstract}

Keywords: Collaborative Knowledge Management, Reference Model, Patterns, Design Process, Project Methodology, Collaborative Cluster, Product Lifecycle Management.

\section{Introduction}

In recent years, tools for integrated information management are aimed at increasing performance in collaborative environments. Specifically, tools like PDM (Product Data Management), cPDm (collaborative Product Definition management) and PLM (Product Lifecycle Management), which allow simplifying and unifying the flows of product information, operating with data from all departments of the company [1]. The fact that the most modern of those tools are Web-compatible also facilitates the inter-enterprise communication, allowing the consideration of the extended enterprise concept. Nevertheless, the implementation of design environments based on these tools is not a trivial question: it requires the introduction of cultural and organizational changes in the enterprise. In this paper we want to emphasize the changes that affect knowledge management (KM), which is defined by Ergazakis et al. [2] as the process of creating value from the intangible assets of an enterprise; it can be related to the internal knowledge of the enterprise, and also to customers and stakeholders. In general, capture, classification, storage and continued update of the knowledge are fundamental actions in every continuous renovation process. In particular, the need to acquire knowledge is critical in new product development [3].

Knowledge management is a particularly important bottleneck for SMEs. When SMEs work in an isolated way, they do not have sufficient economic or technological capacity to promote procedures based on the management of processes addressed to collaborative environments. On the contrary, they do fulfil enough capacity when they work in an Extended Enterprise environment, but in this case, they lack the organizational structure as much as the leadership for driving the process, it is to say, for managing the necessary changes. Besides, it is particularly significant that SME's lack the mutual confidence necessary to share knowledge, and they lack too the experience to implement mechanisms of knowledge management to share knowledge within the Extended Enterprise without running the risk of bringing out all his know-how into the open.

Within this context, the authors are participating in a project (CE-TILE) aimed at establishing a network of knowledge to allow collaborating and sharing information among ceramic tile sector companies (SMEs, most of them), with the aim of solving the knowledge management problems related in general to the product lifecycle processes, and in particular, to the design process. The commercial applications Collaboration Projects and Collaboration cFolders [4] were selected for their utilization within the project. They allow all the extended enterprise partners to join the design process and to share knowledge. But the implementation of these tools is complex: patterns of behaviour that can support the commercial applications, that respond to the roles and tasks of the different members from the extended company, and that guarantee the privacy of the internal knowledge management for each enterprise must be defined. In this sense, this paper tries to show the relevance of the use of patterns to get knowledge management. Next, in section 2, the need to establish patterns that standardize information and knowledge in collaborative work is justified. Section 3 points out the specific aspects in the CE-TILE project development where models or patterns have been used, whereas section 4 presents the use of different types of patterns: the forms for capturing knowledge and the representation techniques to formalize it (4.1.), and the patterns created in the chosen software application for the management of the processes (4.2.). Section 5 presents the results. A section for the conclusions closes this paper. 


\section{About the Need of Reference Models and Patterns for Knowledge Management}

The ceramic tile design process involves different types of companies that work and collaborate together with a common objective: the new product development (NPD). Nevertheless, collaboration is frequently inefficient, as guidelines for a suitable management of relations and processes have not been settled down. It has been argued that initiatives for KM will result in an improvement in the processes execution [5]. Hence, the use of reference models and patterns for the processes and activities is essential, since these processes suppose a critical connection between the KM and the results obtained. Thus, the establishment of reference patterns allows the access and conservation of the possessed knowledge. Instances of certain tasks or processes take place by means of the execution of these patterns, and like this, it is allowed the use of the knowledge [6]. Patterns and models can feed themselves with the experience to improve later execution. In other words, there is a need to establish models and patterns of collaborative knowledge to improve the work and relations among the enterprises implied in the design process. Enterprise modelling, and specifically processes modelling, is an effective tool for the management of organizational changes [7]. Patterns transform implicit knowledge into explicit one, which is the unique one that can be used by all the members of an organization. It is collective knowledge, that is to say, the knowledge found at processes of a work group or organizational unit [8]. Pattern libraries make it easier the work, as they allow both to select the suitable one in each particular case and to update and add new patterns throughout new experiences development. In sum, "creating a knowledge repository, for being understood, used and shared by the partners" is basic [9].

Models and patterns are neither static nor universal; on the contrary, they must be discussed, reviewed, modified, enriched, etc., in a dynamic and collaborative process, until they fit the needs and specific requirements. These patterns and models, combined with new technologies for the communication and the collaboration, which are more and more integrated in the different aspects of the daily work, suppose a powerful strength in the enterprise knowledge architecture, that offers interesting opportunities for the competitive improvement, basically when relations take place at inter-enterprise level [6].

\section{Knowledge Management at CE-TILE Project}

Different models and patterns have been developed in the CE-TILE project: a) a methodology for the project development and certain forms for capturing knowledge, which are based on the MOKA methodology [10] and the KBE lifecycle, and b) activity models for formalising knowledge, and specific patterns for a software application.

The CE-TILE project pursued the development of a specific model for the management of collaborative projects. First, the specific model was obtained from a wider scope reference model. Then, it served as a basis to design a pilot technological infrastructure and a collaborative experience. Thus, several cycles were generated in the CE-TILE project:

1. Definition of a Conceptual Frame (which establishes a common vocabulary and delimits the system reach) and a Generic Activity Model. It constitutes a "should-be" model that represents the ideal activities for a generic design system, pertaining to products marked by tendencies and fashions.

2. Definition of a Specific Reference Model for ceramic product design projects (it is formed by activity, information, organizational, role, and other partial models), and establishment of a technological infrastructure and a software platform for developing a pilot experience (next cycle). Several applications pertaining to MySAP PLM (which was the tool chosen for the accomplishment of the pilot experience) were analysed, and finally, the solution cProjects Suite was applied for the process/project management and for favouring the cooperation. cProjects Suite is formed by the applications Collaboration Projects (cProjects), that gives support to the project management, and Collaboration Folders (cFolders), that consists of a platform for communication and for sharing knowledge among the project participants. The configuration of these applications to adapt them to the ceramic sector characteristics also belongs to this second cycle of the project.

In this cycle, some forms were used for capturing knowledge, and generic patterns were developed for standardizing the types of projects in the software applications.

3. Development of a Pilot Experience that allows valuing the advantages reached by collaborative design environments. Establishment of a Specific Activity Model for the pilot experience ("to-be" model).

The results were a few models of the design process with different coverage and concretion level. The knowhow we gained in the platform design and development, and in the own pilot experience, became the key for the gradual refinement of the specific model. Thus, these three cycles are not sequential, as some information flows exist among them, so that its mutual re-feeding causes the continuous improvement of the process.

For an efficient development of these cycles and their re-feedings, it is necessary to define a methodology that establishes the actions to undertake, and that makes possible its improvement and reusability. A methodology fundamentally consists [11] of a set of instructions and guidelines on how a complex process must be carried 
out. According to Lovett et al. [11], some of the main benefits of the use of a methodology are: to take advantage of the experts knowledge, to avoid the omission of essential tasks, and to easier the project management, since stages and activities can be identified and, if necessary, members of the development equipment can be staffed.

As far as our aim was defining a process to manage the knowledge in the project, we looked for a set of activities which allow the transition between categories of knowledge. Bernus and Kalpic [12] show various knowledge management frameworks and activities. However, the methodology finally chosen for the development of the above defined cycles was based on the generic lifecycle for the development and maintenance of applications KBE, Knowledge Based Engineering. This model of KBE System lifecycle is adopted by the MOKA methodology, aimed at structuring and representing engineering knowledge, giving support to KBE applications [13]. This methodology for analyzing and modelling products, design processes and the related knowledge, requires the previous establishment of the phases of the KBE system lifecycle. These stages fit the actions that configure each cycle of the CE-TILE project: a) Identify, where we established the action plan, the objectives and the scope of the project cycles; b) Justify, to obtain the project establishment and the agreement of the implied parts, c) Capture, in order to acquire, structure, represent (in an informal way) and add knowledge; d) Formalise the knowledge previously captured in the frame of a suitable technique; e) Build; configuring the software tools chosen for the pilot experience, and establishing the operative infrastructure (it corresponds with the Package stage in the model of KBE System Lifecycle), and f) Execute, where the MOKA original actions Distribute, Introduce and Use were summarized in only one, focused on the execution and the performance.

We apply this KBE lifecycle customized to the three stages of development of the CE-TILE project, which go from a generic point of view up to more specific aspects. A singularity is than in the first cycle, the two last actions (Build and Execute) are not considered, because construction and execution make no sense in the generic activity model. The construction of the software system for the improvement in the knowledge interchange begins in the second of the cycles, and the execution corresponds to the third cycle. Although these lifecycles are convergent and go throughout their development towards a more specific level of concretion, they in fact constitute an iterative process of re-feeding, to take advantage of the opportunities to complete and to improve the acquired knowledge. Therefore, sometimes it is worth to return towards levels of a global point of view once we have reached actions pertaining to the most specific cycles. This can happen because it is simpler to define generic aspects once certain specific information has been obtained, or because the opportunity to complete and to improve the knowledge already acquired throughout the project development has been identified. It is indeed what has happened with the generic development, previous to the ceramic specific modelling. Therefore, these cycles allow the continuous improvement of the project, by means of the different directions and senses in which information and knowledge flow between actions.

\section{Use of Patterns at Collaborative Knowledge Management}

As our objective is centred in the analysis of the process-knowledge-management in the ceramic design, we are going to focus our attention on the second of the project cycles, that is to say, the specific one for the ceramic sector development. In this cycle, the actions with a greater prominence in knowledge management agree with those that the MOKA methodology considers to be the main phases [13]: Capture and Formalize. We shall also consider the requirements of the Build stage.

Once the informal representation of the process knowledge has been obtained (Capture), the following step is formal modelling, or formalization (Formalize). The design activities, reflected in an IDEF0 model, are used to define end-to-end processes. Next, the building or implementation of the adapted functionalities (cProjects and cFolders) can begin, and specific patterns for defining and structuring processes to be executed must be created (Build). In the following sections the option for capturing knowledge in the second cycle of the project is explained, as well as the creation of standardized patterns for the application chosen in the pilot experience for managing the project.

\subsection{Knowledge Capture, Storage and Formalization}

In order to obtain the structured management of the acquired knowledge, another adaptation with respect to the MOKA methodology has been used. We use the forms offered by this methodology to capture and informally store knowledge. These forms are denominated ICARE forms, from the different elements or categories in which the knowledge is classified: Illustrations, Constraints, Activities, Rules and Entities [10]. In our case, we have used forms only for two kinds of elements: Activities (for the processes description) and Entities (to detail documents related to the product). The use of these forms has been beneficial, since it has facilitated the work of collecting and retaining information from interviews in a structured way, while it also established the reach of the required knowledge. This second aspect is advantageous as it guides the explanations of the interviewed person. From the information stored in these forms, the formalization in IDEF0 of the activity model has been directly tackled. Some partial IDEF0 diagrams are shown in [14] and [15]. Later, these activities have been represented in "end-to-end" processes (fig.1), which allowed us to obtain a graphical representation of a complete process and detailed knowledge of the activities flows and its 
sequence. Besides, all the responsibilities involved in the process are identified, with the support of roleactivity matrices, including the necessary relations among areas or partners. This technique also facilitates the identification of problems and opportunities of improvement, and has into account the software applications that give support in the project.

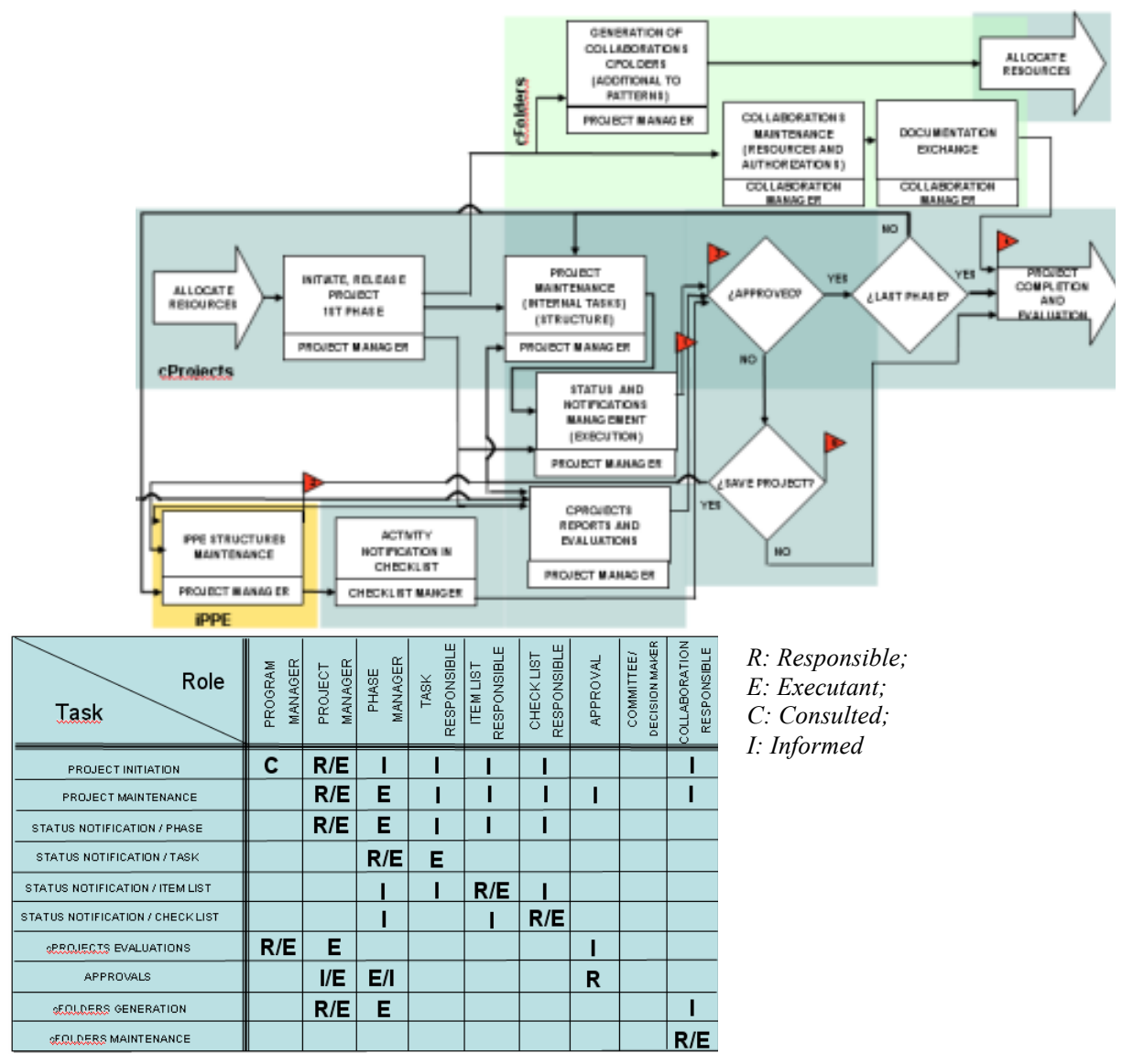

Fig. 1: Formalization of the captured knowledge: "End-to-end" processes, with a matrix of responsibilities

\subsection{Build: Elaboration of Generic Patterns for the Adopted Solution}

Current needs in projects of development require software solutions that offer new functionalities, able to allow a wide reach, including all the processes throughout the product lifecycle. PLM give support to product and process information, through departments and companies in the supply chain. Since our interest is centred in the collaborative knowledge management of the process, we have chosen the solution cProjects Suite of mySAP PLM to support the management of the pilot experience. There are other tools for project management with a larger reach (like Project System, which also belongs to mySAP PLM), but the former applications fit better our interests as they specially emphasize collaboration and communication among enterprises, and consider some aspects of the quality in the process. These are key features for reaching the objectives of the project.

Specifically, cProjects allows the creation of different types of patterns or models, among which project models will be emphasized. This application structures the project in a set of elements, such as phases, tasks, checklists, etc. In this way, a project can be created, and defined like a project model. Thus, a pattern which can be used to facilitate the creation of new projects with similar characteristics and structure is obtained. This way, prior to a new product development process, the project model can be chosen and, if necessary, adapted for its use.

Different types of projects (projects with different structures, intervening roles, terms, objectives, etc.) can exist, due, for instance, to the desired type of product, to the proposal origin, etc. Different options have been analyzed by the CE-TILE research team, to generate project models that constitute a knowledge repository, and that can be used by the ceramic enterprises at new project creation, depending on its type. It is not an automatic selection process; the person in charge of the project will use his/her experience and knowledge to decide, from the models available, the option that better adapts to the particularities of the specific project. One possibility consists of generating a single "comprehensive" model, with a structure that includes all the known project variants, and which will be simplified whenever it is needed to create a new project, eliminating the unnecessary elements. In the pilot experience, the research group established a preliminary generic pattern. The participating enterprises simplified this first pattern for its application. Several phases (like Detailed design), tasks (like Obtain a plaster model of the relief of the model) and items of checklists (like Special enamels test) were eliminated, and other were modified (fig.2). Nevertheless, this option is too complex to be settled like a general norm. For this reason, at the CE-TILE project, the research group should 
identify a standard set of models for different types of projects (based on the information captured at interviews and on the knowledge from literature), so that the model is chosen by the person in charge of each new project, depending on the particular needs (for example, to define a model to develop tile coverings, another one for pavings, etc.) and it is adapted, if required, by creating or eliminating elements. Our pilot experience adds evidences in the sense that having a "standard" set of models, previously elaborated either by a university research group or by an external consultant enterprise, is beneficial for the companies during the process of selection of the appropriate model. In any case, in the enterprises, the people in charge of the project are the responsible in choosing the model that adapts to the characteristics of each particular project. An interesting question that remains open for the future is the search of guided selection methods of the appropriate model for each situation. This set of models can be enlarged gradually with new "tailored" patterns, based on the experience obtained by the ceramic companies in the development of new projects. cFolders also allows the constitution of models to structure folders, documents, forums, and other elements in the collaborations that are created in the project.

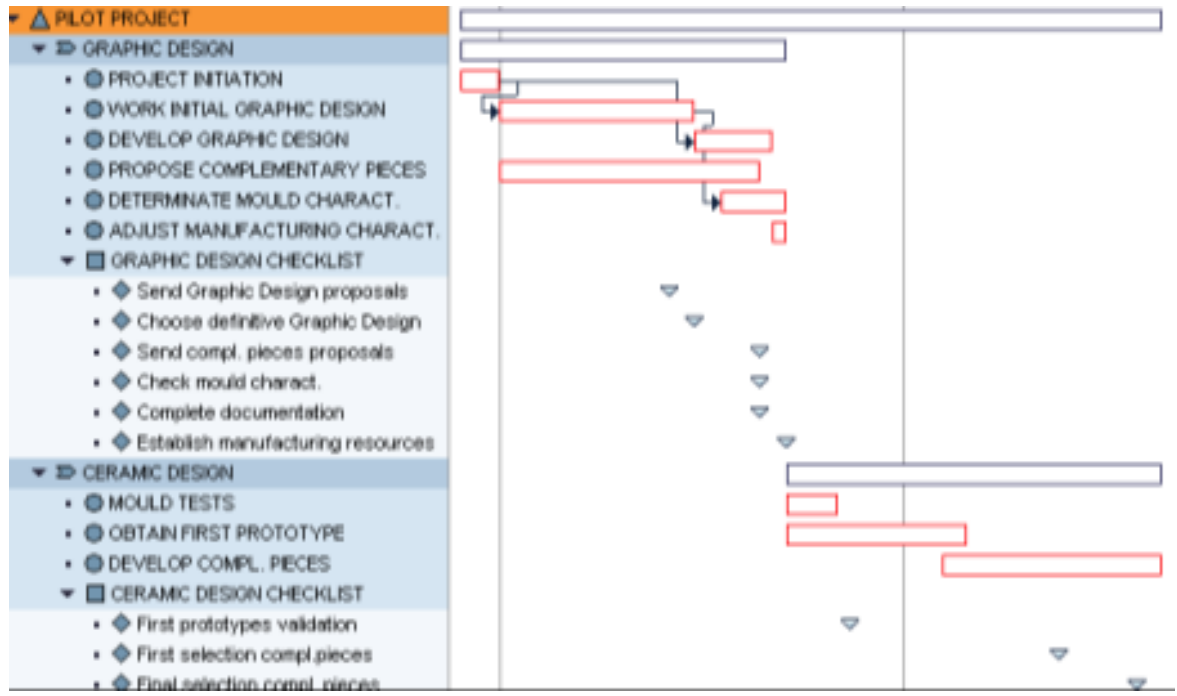

Fig. 2: Structure of project phases, activities and checklists

\section{Results}

Currently we are in the closing phase of the CE-TILE pilot experience, in which the end users of the PLM applications must evaluate the results obtained. The methodology for the project development was initially adopted to have a general guideline. As the project advanced, we saw those cycles to be dynamic, and to have characteristics that facilitate re-feedings, which initially had not been considered. These information flows have allowed us to obtain richer results. With respect to the forms for the knowledge achievement, they have prevented the omission of relevant information. In addition, the professionals interviewed to capture their informal knowledge, did better understood what they were asked, and the resulting formal knowledge was stored in a more structured way. The guided interviews allowed a faster knowledge capture.

The models defined in the application cProjects allow to create projects from patterns previously established. These models were developed by the CE-TILE research group, based on the information provided by the ceramic companies and the knowledge captured from the bibliography. A repository is obtained therefore, of which the people in charge of the NPD project will have to choose the suitable model in each particular case. In the pilot experience, the research group established a generic model, which was later simplified by the ceramic companies, for developing the pilot experience.

This fact aids to standardize the project structure as well as the existing types of projects. In addition, the omission of important aspects is avoided; both internal aspects (like procedures, instructions, rules, etc.) and external ones (norms, legislation, etc.). Anyway, it is important for the projects to adapt to the necessary particular conditions in each case, that is to say, that patterns always suppose an aid and a support for the work, without restricting freedom or creativity. Although the pattern initially developed was quite general, it focused on a certain type of project. It is expected to enrich it with the pilot experience results and to establish new patterns that respond to the needs of new product development projects.

\section{Conclusions}

The creation, storage, adaptation and use of patterns for planning, defining and controlling the execution of processes and activities, constitute a very effective tool in knowledge management. Patterns allow to establish, specify, standardize and apply knowledge, and also to enrich the existing one and to create new knowledge. 
They establish the steps to follow, serving as a guide for the accomplishment of the actions, and at the same time they constitute a method to verify that execution fits planning. They compile knowledge, and feed themselves with new experiences. Like this, they are framed in an iterative process of continuous improvement. Therefore, they are not rigid structures, but they must have a high flexibility.

The article has presented the use of patterns and models in the development of a collaborative project. The development of the pilot experience allowed to identify opportunities for the use of patterns in knowledge management. We understand that an exhaustive analysis of software tools for the management of new product development projects exceeds the reach of the article. We limited the extent of the work to describe the election of collaborative applications in a particular experience, limited by the needs of the participant companies. One of these limitations is that the chosen tool had to belong to SAP, or to a fully compatible solution, since this was the already implemented ERP in one of the leader companies taking part in the pilot experience. Among the tools available in mySAP PLM, the research team analyzed several options for the project management, opting finally for cProjects Suite, since it is characterized by its collaborative nature. This choice responds to the collaboration needs of development projects, in greater extend than other applications of mySAP PLM, as it can be the case of Project System.

It is expected in an immediate future to extend the number of patterns for structuring and characterizing different types of new product development projects, as well as the patterns related to types of collaborations, with the support of the PLM applications. Another interesting aspect for the modelling and the infrastructure improvement, in which the research group is interested, consists of the incorporation of new visions and generic utilities, like a greater consideration of the voice of the customer in the design process, or the aggregation of new quality methodologies. These visions and utilities must validate their suitability and be concreted by means of its incorporation in new and more complete design experiences. The results obtained should extend and improve the existing models.

\section{References}

1. Dickerson C. PDM Product Data Management: An Overview. Society of Manufacturing Engineers Blue Books. CASA.SME. (1997).

2. Ergazakis K., Karnezis K., Metaxiotis K. and Psarras, I. (2002). Knowledge Management in Enterprises: A Research Agenda. PAKM 2002, LNAI 2569, pp.37-48.

3. Peregrim J.T. Knowledge Acquisition in New Product Development. Product Development \& Management Association. http://www.pdma.org/visions/oct96/pe.html

4. SAP AG, "MySAP Product Lifecycle Management. Statement of Direction”, 2005. http://www.sap.com/solutions/business-suite/plm/brochures/index.epx

5. Nickols F. 2000 "Knowlegde Management (KM) and Process Performance. Implications for Action". http://home.att.net/ nickols/KM_and_Processes.htm

6. Manheim M.L. (1998). Beyond Groupware and Workflow (The theory of cognitive informatics and its implication for a people-based enterprise information architecture). Web site http://www.e-workflow.org

7. Chung P.W.H., Cheung L. Stader J., Jarvis P., Moore J. and Macintosh A. (2002). Knowledge-based process management - an approach to handling adaptative workflow. Knowledge-Based systems. 16. pp. 149-160.

8. Mittelmann A. (2002). Practical Aspects of Knowledge Management in Industry. Proceedings 4th International Conference on Practical Aspects of Knowledge Management, Vienna 3 Dec 2002

9. Mo J.P.T. and Zhou M. (2003). Tools and methods for managing intangible assets of virtual enterprise. Computers in industry, 51.pp. 197-210.

10. Stokes M. for MOKA Consortium. (2001). Managing Engineering Knowledge - MOKA Methodology for Knowledge Based Engineering Applications. Professional Engineering Publishing. ISBN: 1860582958.

11. Lovett P.J., Ingram A. and Bancroft, C.N. (2000) "Knowledge-based engineering for SMEs - a methodology". Journal of Materials Processing Technology. 107 pp. 384-389.

12. Bernus P. and Kalpic B. (2004). "The Nature of Knowledge and its Sharing through Models". Knowledge Sharing in the Integrated Enterprise. International Federation for Information Processing. ICEIMT 04 ISBN 0-387-26608-9.

13. Oldham K., Kneebone S., Callot M., Murton A. and Brimble R. (1998) "MOKA - a methodology and tools Oriented to Knowledge-based engineering Applications". Proceedings of the Conference on Integration in Manufacturing. Göteborg, Sweden. pp. 198-207.

14. Vila C., Romero F., Galmés V. and Agost M.J. (2005) "Collaborative Solution for Cooperation, Coordination and Knowledge Management in the Ceramic Tile Design Chain". Proceedings of Second International Conference, Cooperative Design, Visualization, and Engineering. Palma de Mallorca, Spain. LNCS 3675, pp.86-93.

15. Romero F., Company P., Vila C. and Agost M.J. "Enterprise Activity Modelling in a Collaborative Ceramic Tile Design Chain". Research in Engineering Design. Under revision. 